\title{
Die katholische Kirche und die Confessio Augustana
}

\section{Rückblick auf Augsburg 1530 und Vorausblick auf Augsburg 2030}

Im Jahre 2017, 500 Jahre nach 1517, das als Beginn der Reformation in Deutschland gilt, haben wir dieses geschichtlichen Geschehens gedacht, und zwar in ökumenischer Gemeinschaft, vor allem im schwedischen Lund mit der Anwesenheit von Papst Franziskus und dem Präsidenten und Generalsekretär des Lutherischen Weltbundes. ${ }^{1}$ Dieses gemeinsame Reformationsgedenken wäre nicht möglich gewesen ohne die Gemeinsame Erklärung zur Rechtfertigungslehre, die zwischen dem Lutherischen Weltbund und dem Päpstlichen Rat zur Förderung der Einheit der Christen genau vor zwanzig Jahren am 31. Oktober 1999 in Augsburg unterzeichnet worden ist. Nun richtet sich unser Blick wiederum nach Augsburg, indem wir auf den fünfhundertsten Jahrestag des Augsburger Reichstages und der Übergabe des Augsburger Bekenntnisses an Kaiser Karl V. durch evangelische Fürsten und Reichsstadträte am 25. Juni 1530 zugehen.

Dieses Ereignis in ökumenischer Gemeinschaft zu feiern, haben wir noch mehr Grund als im Jahre 2017. Denn zum Reichstag in Augsburg hat Kaiser Karl V. eingeladen, um auf der einen Seite die politischen Kräfte zur Abwehr der großen Türkengefahr im Osten zu sammeln, und um auf der anderen Seite die bedrohte Einheit im Glauben wiederherstellen. Diesbezüglich sollten die Beratungen auf dem Reichstag dem Ziel dienen: „damit durch uns alle eine gemeinsame wahre Religion angenommen und gehalten wird und wir so, wie wir alle unter einem Christus stehen und streiten, auch alle in einer Gemeinschaft und Kirche in Einigkeit leben“. Mit diesen Worten hat der kursächsische Kanzler Brück in der Vorrede zum Augsburger Bekenntnis das Einladungsschreiben von Kaiser Karl V. anklingen lassen, um damit zum Ausdruck zu bringen, dass sich die Teilnehmer am Reichstag dem vom Kaiser anvisierten Ziel der Wiederherstellung der Einheit im Glauben verpflichtet wissen.

1 Vortrag am Internationalen Symposium „Die Confessio Augustana im ökumenischen Gespräch“ an der Europäischen Melanchthon-Akademie Bretten in der Stiftskirche Bretten am 12. Oktober 2019.

Ә OpenAccess. () 2022 Kurt Kardinal Koch, publiziert von De Gruyter. (cc))BY-NC-ND Dieses Werk ist lizenziert unter einer Creative Commons Namensnennung - Nicht kommerziell - Keine Bearbeitung 4.0 International Lizenz. https://doi.org/10.1515/9783110683868-029 
Wenn man diese Zielbestimmung bedenkt, kann man den Reichstag zu Augsburg in seiner ökumenischen Bedeutung nicht unterschätzen. Der Ökumenische Arbeitskreis evangelischer und katholischer Theologen hat deshalb mit Recht geurteilt: „Möglicherweise waren die Kirchen der abendländischen Christenheit in der Tat beim Reichstag zu Augsburg 1530 einander so nahe wie später nie wieder." ${ }^{2}$ Wenn man diese historische und ökumenische Bedeutung bedenkt, kann man nur hoffen, dass der fünfhundertste Gedenktag des Reichtags zu Augsburg und der damals verkündeten Confessio Augustana in zumindest ebenso intensiver ökumenischer Gemeinschaft wie das Reformationsgedenken im Jahre 2017 begangen werden wird.

Darauf müssen wir zurückkommen. Zuvor jedoch muss kurz in Erinnerung gerufen werden, dass zwischen dem Beginn der Reformation und dem Reichstag zu Augsburg schwerwiegende Ereignisse stattgefunden haben, nämlich die Veröffentlichung der Bannandrohungsbulle Exsurge Domine vom 15. Juni 1520, mit der Papst Leo X. 41 Sätze aus den Schriften Luthers als „häretisch oder skandalös oder falsch oder beleidigend für fromme Ohren oder die einfachen Gemüter verführend und der katholischen Wahrheit entgegenstehend“ verurteilt hat, als Luthers Reaktion darauf seine Verbrennung der Bannandrohungsbulle vor dem Elstertor in Wittenberg am 10. Oktober 1520 und die Veröffentlichung der Bulle Decet Romanum Pontificem vom 3. Januar 1521, mit der Luther und alle, die ihm folgen und ihn schützen, als Häretiker und zu meidende Exkommunizierte erklärt worden sind, und schließlich die Verhängung der Reichsacht über Luther und seine Anhänger im Wormser Edikt.

Auf diesem Hintergrund leuchtet die besondere geschichtliche Bedeutung des Reichstags zu Augsburg erst recht ein. Denn zunächst waren die Vorzeichen dafür nicht positiv, zumal vor allem der katholische Theologe Johannes Eck sich für eine öffentliche Disputation über die Religionsfrage stark gemacht und dazu seine 404 Artikel gegen die Häretiker veröffentlicht hat, und zumal von anderer Seite her auf die Durchführung des Wormser Edikts gedrängt worden ist, was jedoch politisch nicht durchzusetzen gewesen ist. Gegenüber der Option der Entscheidung der Religionsfrage durch eine Disputation und gegenüber dem Versuch, die lutherischen Prediger als Häretiker zu erweisen, hat man sich schließlich auf dem Reichstag zu Augsburg für ein freundschaftliches Gespräch

2 V. Leppin/D. Sattler (Hg.), Reformation 1517-2017. Ökumenische Perspektiven, Freiburg i.B./ Göttingen 2014, 67. 
entschieden und dazu einen Ausschuss nominiert, der aus „sachverständigen und zu Frieden und Einigkeit geneigten“ Personen bestehen soll. ${ }^{3}$

Als Grundlage für dieses amicum colloquium hat das Augsburger Bekenntnis gedient, das am Reichstag offiziell dem Kaiser übergeben worden ist. Dabei ist der vorbereiteten sächsischen Verteidigung der von lutherischer Seite geänderten Kirchenbräuche eine Zusammenfassung der Glaubensartikel vorangestellt worden, um so zu bekunden, dass in den evangelischen Gemeinden nicht Sonderlehren vertreten werden und auch nicht eine neue Kirche gegründet werde, dass es vielmehr um die Erneuerung und dazu notwendige Reinigung des christlichen Glaubens, jedoch in Einklang mit der Alten Kirche und auch mit der Römischen Kirche gehe.

Das Augsburger Bekenntnis ist wesentlich dem unermüdlichen Bemühen des bedeutenden Theologen und engagierten Reformators Philipp Melanchthon zu verdanken, ${ }^{4}$ der überzeugt gewesen ist, dass die Erneuerung der Kirche und das Festhalten an ihrer Einheit unlösbar zusammengehören. Er hat sich damit als großer „Ökumeniker seiner Zeit“ erwiesen, „der - unter den damaligen historischen Voraussetzungen - die allerletzten Möglichkeiten für die Bewahrung der Einheit der Kirche auszuloten suchte“ ${ }^{\text {*5 }}$. Das von ihm verfasste Augsburger Bekenntnis ist deshalb kein Dokument der Spaltung, sondern des entschiedenen Willens zur Versöhnung und zur Bewahrung der Einheit und des Friedens. Man kann deshalb von einem katholischen Dokument reden, weil es sich in Kontinuität mit der Alten Kirche und auch mit der Römischen Kirche versteht, und man kann von einem ökumenischen Dokument reden, weil es nicht das Bekenntnis einer partikularen Konfessionskirche sein will, sondern den Anspruch erhebt, dass es in ihm um die eine, heilige, katholische und apostolische Kirche geht und dass sie in ihm spricht.

3 Vgl. V. Pfnür, Excommunicatio und amicum colloquium. Das Religionsgespräch auf dem Reichstag zu Augsburg 1530 auf dem Hintergrund der Frage des Lutherbannes, in: W. Beinert/K. Feiereis/H.-J. Röhrig (Hg.), Unterwegs zum einen Glauben. Festschrift für Lothar Ulrich zum 65. Geburtstag, Leipzig 1997, 448-460.

4 Vgl. G. Frank (Hg.), Der Theologe Melanchthon, Stuttgart 2000; J. Haustein (Hg.), Philipp Melanchthon. Ein Wegbereiter für die Ökumene, Göttingen 1997.

5 W. Thönissen, Gerechtigkeit oder Barmherzigkeit? Das ökumenische Ringen um die Rechtfertigung, Leipzig/Paderborn 2016, 138. 


\section{Die ökumenische Intention der Confessio Augustana}

„Die Gemeinden lehren bei uns in voller Übereinstimmung“: Mit diesem Satz, der über dem ersten Artikel steht, jedoch für das ganze Bekenntnis gilt, wird das Grundanliegen des Augsburger Bekenntnisses - gleichsam wie der Notenschlüssel der ganzen Melodie - zum Ausdruck gebracht, dass es den katholischen Glauben bezeugen und in diesem Sinn ein katholisches Bekenntnis sein will. Diese Überzeugung wird im Text gleich dreimal, und zwar keineswegs beiläufig, sondern an wichtigen Knotenpunkten formuliert. Beim Abschluss des ersten Teils wird festgehalten: „Das ist ungefähr die Summe der Lehre auf unserer Seite. Es zeigt sich, dass nichts darin vorhanden ist, was abweicht von der Heiligen Schrift und von der allgemeinen und von der römischen Kirche, wie wir sie aus den Kirchenschriftstellern kennen. Infolgedessen ist das Urteil derer ungerecht, welche die Unsrigen für Ketzer gehalten wissen sollen.“ Gleich zu Beginn des zweiten Teils wird nochmals betont, dass ,in keinem Glaubenssatz von der allgemeinen Kirche“ abgewichen werde, sondern dass „nur einige wenige Missbräuche“ beseitigt werden, „welche neu und gegen die Absicht der Kirchengesetze im Laufe der Zeiten zu Unrecht eingeführt wurden“. Und am Schluss des Dokumentes wird nochmals zusammenfassend beteuert: „Bei uns gilt weder in der Lehre noch in den Zeremonien etwas, was der Heiligen Schrift oder der allgemeinen Kirche entgegensteht. Denn es liegt klar zutage, wie sorgfältig wir uns gehütet haben, dass sich nicht neue und gottlose Glaubenssätze bei uns in die Kirche einschlichen.“

Diese Überzeugung kommt somit deutlich zum Ausdruck in der Zweiteilung des Bekenntnisses. Denn das Hauptanliegen der evangelischen Seite bestand darin, die Abschaffung von kirchlichen Bräuchen und Ordnungen zu rechtfertigen, die die Evangelischen als Missbräuche, jedoch nicht als kirchentrennende Fragen beurteilt haben. Diese Ausführungen bilden den zweiten Teil des Bekenntnisses, der seinerseits die Torgauer Artikel vom 27. März 1530 enthält, mit denen sich die Wittenberger Theologen auf Augsburg vorbereitet haben. Im Blick auf die von den Evangelischen vorgenommenen Änderungen wird beansprucht, dass sie in Übereinstimmung mit „Gottes Gebot“ vorgenommen worden sind, nämlich die Austeilung des Abendmahls in beiden Gestalten, die Einführung der Priesterehe, die Reform der Messliturgie, die Nicht-Notwendigkeit des Nennens aller Sünden in der Beichte, die Abschaffung der Fastengebote, die Aufhebung der Klostergelübde und die Beschränkung der Autorität der Bischöfe auf ihre geistliche Vollmacht. 
Der erste Teil des Bekenntnisses über die grundlegenden Glaubensartikel ist demgegenüber ursprünglich nicht vorgesehen gewesen, sondern ist erst in Augsburg hinzugefügt worden, um zu dokumentieren, dass die evangelischen Gemeinden mit ihren grundlegenden Glaubensartikeln mit den Lehrfundamenten der Alten Kirche übereinstimmen. Die Glaubensartikel werden dabei vor allem in drei Themenkreisen dargestellt: Die Artikel 1 bis 3 über Gott, den Sohn Gottes und die Erbsünde knüpfen bewusst an die dogmatische Tradition der Kirche an und rezipieren die altkirchlichen Lehrentscheidungen, wobei freilich der Artikel über die Erbsünde sowohl durch seine Anordnung wie seinen Inhalt eine sehr eigene Färbung aufweist. Artikel 4 bis 6 kreisen um das Herzstück der Reformation, nämlich das Evangelium von der Rechtfertigung, das Wirken des Heiligen Geistes im Wort und in den Sakramenten und im Predigtamt und über die Früchte des Glaubens, nämlich das innere „Muss“ der guten Werke, das nochmals in Artikel 20 aufgegriffen und ergänzt wird mit den Artikeln über den freien Willen und den Ursprung der Sünde. Im dritten Kreis in den Artikeln 7-15 geht es um ekklesiologische Themen, was die Kirche ist und worin ihre Einheit besteht, über die Sakramente der Taufe, des Abendmahls, Beichte und Buße und über die Bedeutung und den Gebrauch der Sakramente, über Amt und Ordination und die kirchlichen Ordnungen. $\mathrm{Zu}$ diesen drei Kreisen fügen sich noch einzelne Artikel an über das Verhältnis des Christen zu den weltlichen Angelegenheiten, die Wiederkunft Christi und die Heiligenverehrung.

Mit dieser Zweiteilung im Bekenntnis soll die Überzeugung artikuliert werden, dass die Evangelischen den Glauben der Katholischen Kirche teilen, dass der Unterschied nur in den so genannten Missbräuchen liegt, die in den evangelischen Gemeinden abgeschafft worden sind. Man wird freilich urteilen müssen, dass diese Zweiteilung nicht in konsequenter Weise durchgehalten ist, wie nur kurz an wenigen Beispielen verdeutlicht werden kann. Der Artikel über die Messe befindet sich im zweiten Teil, weil die von den Reformatoren kritisierte Entwicklung zur Primatmesse des Priesters als abusus bezeichnet und mit dem Stipendienwesen begründet wird. Dann jedoch wird behauptet, die Vermehrung der Privatmessen sei in der Lehre begründet, Christus habe mit seinem Leiden Genugtuung nur für die Erbsünde geleistet, während für die übrigen Sünden die Messe als Opfer eingesetzt worden sei. Während hier somit ein ernsthaftes theologisches Problem behandelt wird, nimmt sich der Artikel über das Mahl des Herrn im ersten Teil äußerst gedrängt aus. In ähnlicher Weise wird das Mönchtum aus theologischen Gründen abgelehnt, obwohl sich der Artikel im zweiten Teil befindet. Umgekehrt wird über die Heiligenverehrung im ersten Teil gehandelt, wobei vor allem die Anrufung der Heiligen als Missbrauch verurteilt wird.

Auch wenn der Aufbau des Bekenntnisses einige Fragen hinsichtlich seiner Kohärenz aufwirft und auch wenn Realitäten im Bekenntnis als usus, bezie- 
hungsweise abusus bezeichnet werden, die in katholischer Sicht Teil der Glaubenslehre sind, bleibt die Feststellung bestehen, dass das Augsburger Bekenntnis wie wohl kein anderes sowohl in Inhalt als auch Struktur den ökumenischen Willen und die katholische Intention der Reformation Martin Luthers widerspiegelt und dass es aus dem Leben und der Lehre der Katholischen Kirche erwachsen ist, wie der jüngst verstorbene katholische Ökumeniker und Bischof Paul-Werner Scheele mit der ihm eigenen Bildersprache formuliert hat:

Artikel um Artikel erweist sich, dass die CA in katholischem Boden verwurzelt ist, dass sie aus ihm erwachsen ist und mit ihm verbunden bleibt. Sie nimmt nicht von ihm Ausgang im Sinne eines Abgangs, sie ist also nicht einer Rakete zu vergleichen, die irgendwo startet, um dann ganz anderen Gesetzen Folge zu leisten. Bis zur Stunde gibt es ein vielfältig verschlungenes Wurzelgeflecht, das die Elemente des lutherischen Grundbekenntnisses mit Elementen der katholischen Kirche verknüpft. ${ }^{6}$

\section{Anerkennung der Confessio Augustana als katholisches Bekenntnis?}

Von der Katholizität des Augsburger Bekenntnisses ist bereits in der Vergangenheit die Rede gewesen. Vor allem Friedrich Heiler hat schon zu Beginn der Ökumenischen Bewegung anlässlich des vierhundertjährigen Jubiläums der Confessio Augustana im Jahre 1930 auf ihren katholischen Inhalt und ihre katholische Intention hingewiesen und dieses Bekenntnis zur Magna Charta der Katholizität erklärt. ${ }^{7}$ Das ökumenische Interesse an der Confessio Augustana ist sodann in den siebziger Jahren von der katholischen Theologie in Deutschland ausgegangen, vor allem vom damaligen Dogmatiker Joseph Ratzinger und seinem Schüler Vinzenz Pfnür. Joseph Ratzinger hat in seinem viel beachteten Vortrag über die Zukunft des Ökumenismus in Graz im Jahre 1976 auf die ökumenischen Bemühungen hingewiesen, „eine katholische Anerkennung der Confessio Augustana oder richtiger: eine Anerkennung der CA als katholisch zu erreichen und damit die Katholizität der Kirchen Augsburgischen Bekenntnisses festzustellen, die eine korporative Vereinigung in der Unterschiedenheit möglich macht“8. In derselben Sinnrich-

6 P.-W. Scheele, Die Confessio Augustana im Kontext katholischen Lebens und Lehrens, in: H. Fries u. a. (Hg.), Confessio Augustana. Hindernis oder Hilfe?, Regensburg 1979, 207-239, zit. 211. 7 F. Heiler, Die Katholizität der Augustana, in: Die Hochkirche. Monatsschrift der hochkirchlichen Vereinigung e. V. (1930), 4-40.

8 J. Kardinal Ratzinger, Die ökumenische Situation - Orthodoxie, Katholizismus und Reformation, in: ders., Theologische Prinzipienlehre. Bausteine zur Fundamentaltheologie, München 
tung hat Vinzenz Pfnür geurteilt, dass die Confessio Augustana „keine kirchentrennenden Lehren“ vertritt und deshalb „als Zeugnis gemeinchristlichen Glaubens von katholischer Seite bejaht werden kann“9; dieses Urteil hatte er bereits im Blick auf die Rechtfertigungslehre des Bekenntnisses in seiner Dissertation ausführlich begründet. ${ }^{10}$

Nachdem sich auch das Einheitssekretariat in Rom mit der Frage einer möglichen Anerkennung des Augsburger Bekenntnisses als einer legitimen Ausprägung der christlichen Wahrheit beschäftigt und nachdem der Lutherische Weltbund auf seiner Vollversammlung in Daressalam im Juni 1977 diese ökumenische Frage mit einer eigenen Erklärung begrüßt hat, sind vielfältige Bemühungen unternommen worden, um anlässlich des vierhundertfünfzigsten Jahrestags der Confessio Augustana ,ihre katholische Anerkennung“ aussprechen zu können. ${ }^{11}$ Dazu ist vor allem ein gemeinsamer katholisch/lutherischer Interpretationsversuch unternommen worden, mit dem die einzelnen Artikel des Bekenntnisses auf ihre Konsensfähigkeit überprüft worden sind. Diese gemeinsame Untersuchung von lutherischen und katholischen Theologen hat dabei das erfreuliche Ergebnis erbracht, „dass die Confessio Augustana nicht nur die Intention hatte, den gemeinsamen katholischen Glauben zu bezeugen, sondern dass ihre inhaltlichen Aussagen tatsächlich in hohem Masse als Ausdruck dieser Katholizität verstanden werden müssen“. Es ist freilich auch festgestellt worden, dass „noch offene Fragen“ bleiben und „wir die Confessio Augustana nicht als gemeinsames Bekenntnis dieses einen katholischen Glaubens sprechen können“, dass wir aber hoffen, „dass unsere Kirchen Formen finden, diese erkannte Ge-

1982, 203 - 214, zit. 212, jetzt in: ders., Kirche - Zeichen unter den Völkern = Gesammelte Schriften Bd. 8/2, Freiburg i.B. 2010, 717-730, zit. 728.

9 V. Pfnür, Anerkennung der Confessio Augustana durch die katholische Kirche?, in: Internationale katholische Zeitschrift 4 (1975), 298-307.

10 V. Pfnür, Einig in der Rechtfertigungslehre? Die Rechtfertigungslehre in der Confessio Augustana (1530) und die Stellungnahme der katholischen Kontroverstheologie zwischen 1530 und 1535, Wiesbaden 1970.

11 Vgl. Fries u. a., Hindernis oder Hilfe?; B. Lohse/O. H. Pesch (Hg.), Das Augsburger Bekenntnis von 1530 damals und heute, München/Mainz 1980; H. Meyer/H. Schütte/H.- J. Mund (Hg.), Katholische Anerkennung des Augsburgischen Bekenntnisses. Ein Vorstoss zur Einheit zwischen katholischer und lutherischer Kirche, Frankfurt a. M. 1977. Vgl. auch K. Koch, Die Confessio Augustana - Ein katholisches Bekenntnis?, in: ders., Gelähmte Ökumene. Was jetzt noch zu tun ist, Freiburg i.B. 1991, 65-106. 
meinsamkeit anzuzeigen als Zeichen und Hilfe für unsere Gemeinden und vor der Welt"12.

\subsection{Inhaltliche Probleme}

$\mathrm{Zu}$ einer katholischen Anerkennung der Confessio Augustana ist es im Jahre 1980 nicht gekommen, und zwar aus verschiedenen Gründen. Die Schlüsselfrage dürfte dabei diejenige gewesen sein, was unter „Anerkennung“ genauer zu verstehen ist. Eine erste Schwierigkeit ergibt sich bereits von daher, dass die Confessio Augustana nicht nur im zweiten, sondern auch im ersten Teil auch sehr zeitbedingte und obsolet gewordene Aussagen enthält. Wenn es beispielsweise in Artikel 16 heißt, dass es den Christen erlaubt ist, „die Todesstrafe nach dem Recht zu verhängen“, kann man nur hoffen, dass weder katholische noch evangelische Christen eine solche Aussage in der heutigen Zeit ,anerkennen“, in der die Todesstrafe bei uns glücklicherweise abgeschafft worden ist und Papst Franziskus sie prinzipiell geächtet hat. Ebenso entsprechen die harten Urteile über die Wiedertäufer in der Confessio Augustana nicht mehr den heutigen ökumenischen Gesprächen mit diesen anderen reformatorischen Gemeinschaften.

Zweitens wird man von der Katholischen Kirche nicht erwarten können, dass sie den zweiten Teil des Augsburgischen Bekenntnisses heute „anerkennt“. Sie kann und muss gewisse Aussagen wohl historisch anerkennen und geschehene Missbräuche in der Katholischen Kirche in der Vorreformationszeit als Fehlentwicklungen beurteilen und bedauern. Doch nicht alles, was in der Confessio Augustana als Missbräuche beurteilt wird, sind in katholischer Sicht solche. Das Halten gewisser Tage, Fasten, Wallfahrten und das christozentrische Rosenkranzgebet sind nicht „kindische und unnötige Werke“, wie es in Artikel 20 heißt, sondern legitime Frömmigkeitsformen, die auch heute, teilweise auch von evangelischen Christen, geübt werden. Auch über das Mönchtum werden in der Confessio Augustana polemische Pauschalurteile gefällt, die heute schlicht nicht mehr aufrechterhalten werden können, zumal auch in den evangelischen Kirchen geistliche Gemeinschaften wieder heimisch geworden sind, so dass beispielsweise die VELKD im Jahre 1976 in einem Wort an die Gemeinden empfohlen hat, „beim Nachdenken über kirchliche Erneuerung die Kommunitäten als mögliche Gestaltungen christlichen Lebens zu bejahen“"13.

12 H. Meyer/H. Schütte (Hg.), Confessio Augustana. Bekenntnis des einen Glaubens. Gemeinsame Untersuchung lutherischer und katholischer Theologen, Paderborn/Frankfurt a. M. 1980, 333 und 337.

13 In: L. Mohaupt (Hg.), Modelle gelebten Glaubens, Hamburg 1976, 142-144. 
Die Katholische Kirche hat sich zudem sowohl beim Konzil von Trient als auch und vor allem beim Zweiten Vatikanischen Konzil einer tiefgreifenden Erneuerung, freilich in bleibender Kontinuität mit der grossen Tradition, unterzogen, so dass sie sich in den meisten Behauptungen im zweiten Teil der Confessio Augustana nicht mehr wiederfinden kann. Die Gemeinsame Römisch-katholische/Evangelisch-lutherische Kommission hat bereits im Jahre 1980 festgestellt, dass sich im Blick auf die angesprochenen Kritikpunkte „im Leben und Urteil unserer Kirchen Wandungen vollzogen“ haben, „welche die im Augsburgischen Bekenntnis ausgesprochene harte Kritik im Wesentlichen gegenstandslos machen“14. Der ehemalige lutherische Bayerische Landesbischof Hermann Dietzfelbinger hat im Blick auf seine Kirche sogar die Aussage gewagt, dass wir „ehrlicherweise nicht um die Feststellung“ herumkommen,

dass die Augsburgische Konfession in manchem ihrer Abschnitte heute fast eher zur Frage an die Kirche wird, die sich nach ihr nennt, als zu einer Polemik gegen die damalige römischkatholische Kirche. Dass z. B. die Messe, d. i. der eucharistische Gottesdienst, <ohne Ruhm zu reden, bei uns mit grösserer Andacht und Ernst gehalten wird denn bei den Widersachern>, wie es in Artikel XXIV der Augsburgischen Konfession heisst, lässt sich heute nicht behaupten. ${ }^{15}$

Auch was eine mögliche Anerkennung der Glaubensartikel im ersten Teil der Confessio Augustana betrifft, muss ich es mit wenigen Hinweisen bewenden lassen. Wie bereits angesprochen, bestehen diesbezüglich noch offene Fragen, die weiter bearbeitet werden müssen, und Fragen, die in der Confessio Augustana ausgeklammert worden sind, obwohl sie damals als kontrovers betrachtet worden sind, wie beispielsweise die Zahl der Sakramente und das Thema des Fegefeuers. Auch was spezifische Aspekte der episkopalen Verfassung der Kirche und eines Lehramts in ihr betrifft, verhält sich die Confessio Augustana recht schweigsam. Ebenso vermisst man im Kontext der Thematisierung der Katholizität der Kirche eine Behandlung der Frage des Papsttums. Diesbezüglich haben Harding Meyer und Heinz Schütte mit Recht festgestellt: „Dass nach katholischer Auffassung das Petrusamt als Dienst an der Einheit der Gesamtkirche, mit dem der Bischof von Rom betraut

14 Alle unter einem Christus. Stellungnahme der Gemeinsamen Römisch-katholischen/Evangelisch-lutherischen Kommission zum Augsburgischen Bekenntnis, 1980, Nr. 19, in: Dokumente wachsender Übereinstimmung. Sämtliche Berichte und Konsenstexte interkonfessioneller Gespräche auf Weltebene. 1931-1982, hg.v. H. Meyer/H.-J. Urban/L. Vischer, Paderborn/Frankfurt a.M. $1983,323-328$, zit. $326-327$.

15 H. Dietzfelbinger, Schwierigkeiten einer katholischen Anerkennung des Augsburgischen Bekenntnisses aus lutherischer Sicht, in: Meyer/Schütte/Mund, Katholische Anerkennung, 54-59, zit. 58f. 
ist, zur Katholizität der Kirche hinzugehört, eröffnet einen Fragenkomplex, der bekanntlich in der CA nicht direkt angesprochen, vermutlich sogar bewusst ausgeklammert ist." ${ }^{16}$ Dieses Desiderat hat Philipp Melanchthon freilich auf Verlangen der evangelischen Reichsstände später nachholen müssen mit seinem recht pointierten Traktat über die Gewalt und Obrigkeit des Papstes De potestate et primatu papae $^{17}$, der zudem auf der Versammlung des Schmalkaldischen Bundes im Jahre 1537 offiziell zur Confessio Augustana hinzugefügt worden ist.

\subsection{Formale Fragestellungen}

An dieser Stelle geht die inhaltliche Problematik des Begriffs der Anerkennung in seine formale Fragestellung über. Denn nach Augsburg ist die Entwicklung weitergegangen, so dass die Confessio Augustana nur noch eine Bekenntnisschrift neben verschiedenen anderen darstellt, in denen aufgrund einer veränderten historischen Situation der polemische Ton gegen die Katholische Kirche wiederum sehr viel stärker zu vernehmen ist. Bereits die Apologie der Confessio Augustana, die auf die Confutatio Pontificia antwortet, ${ }^{18}$ hat neue dogmatische Grenzlinien gezogen und den Konsens von Augsburg bei vielen Glaubensthemen wieder in Frage gestellt. Dies trifft noch mehr bei Melanchthons Traktat über die Gewalt und Obrigkeit des Papstes zu, der freilich seinerseits viel versöhnlicher erscheint als die zur gleichen Zeit erschienenen Schmalkaldischen Artikel, in denen der Papst als verum Antichristum („Endechrist oder Widerchrist“) bezeichnet wird ${ }^{19}$ und eine Verständigung mit der römischen Kirche als endgültig unmöglich beurteilt wird, weil der Dissens im Grundsätzlichen bestehe.

Von daher stellt sich die Frage, welche Verbindung zwischen der Confessio Augustana, die als der älteste ins Corpus der evangelischen Bekenntnisschriften aufgenommene Selbstausdruck des reformatorischen Glaubensverständnisses gilt, und den weiteren Bekenntnisschriften besteht und welcher Stellenwert ihr in ihnen zugewiesen wird. Bildet die Confessio Augustana für die Lutherischen Kirchen den

16 H. Meyer/H. Schütte, Die Auffassung von Kirche im Augsburgischen Bekenntnis, in: dies., Bekenntnis des einen Glaubens, 168-197, zit. 174.

17 In: Die Bekenntnisschriften der evangelisch-lutherischen Kirche, herausgegeben im Gedenkjahr der Augsburgischen Konfession 1930, Göttingen ${ }^{7} 1976,469-498$.

18 Vgl. H. Immenkötter, Der Reichstag zu Augsburg und die Confutatio, Münster 1979 (Katholisches Leben und Kirchenreform im Zeitalter der Glaubensspaltung 39); E. Iserloh (Hg.), Confessio Augustana und Confutatio. Der Augsburger Reichstag 1530 und die Einheit der Kirche, Münster 1980 (Reformationsgeschichtliche Studien und Texte 118).

19 Artikel christlicher Lehre, in: Bekenntnisschriften, 405-468, zit. 430. 
Grundlagentext, dem größeres Gewicht beigemessen wird als den späteren Bekenntnisschriften, die insofern als Weiterentwicklungen, Kommentierungen und Präzisierungen der Confessio Augustana zu betrachten, aber von ihr her auszulegen sind? Oder ist die Confessio Augustana von den späteren Bekenntnisschriften her zu interpretieren, in denen zum Ausdruck gebracht worden ist, was in Augsburg in diplomatischer Rücksicht im Blick auf das Gelingen des Einigungsversuchs noch nicht gesagt werden, jetzt aber mit klarer Eindeutigkeit zum Ausdruck gebracht werden konnte und musste? Es versteht sich von selbst, dass diese Alternative nur auf der lutherischen Seite entschieden werden kann. Es versteht sich aber ebenso von selbst, dass der Inhalt des Begriffs der „Anerkennung“ wesentlich vom Ergebnis einer solchen verbindlichen Entscheidung abhängt.

Eine weitere Problemstellung, die für die Beantwortung der Frage der „Anerkennung“ der Confessio Augustana von katholischer Seite von Bedeutung ist, betrifft die Beurteilung des Verhältnisses zwischen den Lehraussagen des Augsburger Bekenntnisses zur Theologie Martin Luthers. Diese Frage stellt sich bereits von daher, dass im Kontext der Diskussionen um eine katholische Anerkennung der Confessio Augustana in den siebziger Jahren nicht nur auf evangelischer Seite, sondern auch in der katholischen Lutherforschung verschiedentlich der Verdacht geäußert worden ist, bei diesem Projekt würde eine ökumenische Einigung zwischen der Lutherischen Reformation und Rom ,auf Kosten Martin Luthers“ angestrebt. ${ }^{20}$ Ein solcher Verdacht geht jedoch, so scheint mir, an der eigentlichen Fragestellung vorbei. Denn die Confessio Augustana hat in der lutherisch-katholischen Ökumene nicht deshalb eine besondere Bedeutung, weil sie von Philipp Melanchthon verfasst worden ist, sondern weil es sich um einen verbindlichen Bekenntnistext der evangelischen Gemeinschaft handelt.

Auch im Blick auf das Verhältnis zwischen der Confessio Augustana und der Theologie Luthers stehen wir vor einer Alternative: Wird auf evangelischer Seite im theologischen Werk Martin Luthers die eigentliche Grundlegung der Reformation gesehen, so dass von ihm her die Bekenntnisschriften $\mathrm{zu}$ interpretieren sind? Dann bestünden schwierig zu lösende Differenzen zur Confessio Augustana, zumal wenn man an Luthers reformatorische Programmschriften aus dem Jahre 1520 denkt: An den christlichen Adel deutscher Nation von des christlichen Standes Besserung $^{21}$ und De captivitate Babylonica ecclesiae ${ }^{22}$, mit denen er die sakramentale Ordnung der Katholischen Kirche, jedenfalls in der Weise, in der sie ihm

20 Vgl. P. Manns, Zum Vorhaben einer katholischen Anerkennung der Confessio Augustana: Ökumene auf Kosten Martin Luthers, in: Ökumenische Rundschau 26 (1977), 426 - 450.

21 M. Luther, WA 6, 381-469.

22 M. Luther, WA 6, 497-573. 
damals begegnet ist, verworfen hat und im Blick auf die Kardinal Walter Kasper mit Recht geurteilt hat, mit diesen Schriften habe Luther „einen Bruch mit dem katholischen Kirchenverständnis“ eingeleitet, ${ }^{23}$ der eben in der Confessio Augustana nicht zu finden ist.

Wird jedoch umgekehrt das Augsburgische Bekenntnis als Basis für die ökumenische Verständigung zwischen den Lutherischen Kirchen und der Römischkatholischen Kirche genommen, stellt sich die Frage, wie das Verhältnis zwischen dem theologischen Werk eines einzelnen Theologen und einer kirchlichen Lehraussage zu sehen und zu verwirklichen ist. In katholischer Sicht kann es jedenfalls nicht genügen, wenn bei ökumenischen Dialogen, die verbindliche Entscheidungen von Kirchengemeinschaften vorbereiten sollen, die Position eines einzelnen Theologen - und sei es selbst diejenige des Reformators Martin Luther als Basis genommen wird; die Grundlage muss vielmehr in den kirchlichen Bekenntnisschriften gesehen werden. Deshalb braucht es eine Verständigung darüber, welchen Stellenwert ein kirchliches Bekenntnis in der betreffenden Kirchengemeinschaft innehat. Denn von dieser Klärung hängt wiederum ab, wie die Anerkennung dieses Bekenntnisses vonseiten einer anderen Kirchengemeinschaft zu vollziehen ist. In diesem Sinn hat Kardinal Joseph Ratzinger in den früheren diesbezüglichen Diskussionen betont, dass eine katholische Anerkennung des Augsburgischen Bekenntnisses ihre evangelische Anerkennung voraussetzt, genauer die Anerkennung dessen, dass hier Kirche als Kirche lehren kann und lehrt: „Die evangelische <Anerkennung> wäre in jedem Fall die erste innere Voraussetzung einer katholischen Anerkennung und zugleich ein geistlicher Vorgang, der ökumenische Realität schaffen würde.“24

\section{Bekenntnis der Einheit oder Dokument der Spaltung?}

Von daher stellt sich zugespitzt die Frage nach Autorität und Verbindlichkeit der Confessio Augustana in den Kirchengemeinschaften und im ökumenischen Gespräch. Diese Frage lässt sich aber nicht beantworten, ohne Rechenschaft darüber abzulegen, was zwischen Augsburg 1530 und heute geschehen ist. Denn die Geschichte ist nach Augsburg weitergegangen; und da das Einigungsprojekt in

23 W. Kardinal Kasper, Martin Luther. Eine ökumenische Perspektive, Ostfildern 2016, 31.

24 J. Kardinal Ratzinger, Klarstellungen zur Frage einer <Anerkennung> der Confessio Augustana durch die katholische Kirche, in: ders., Theologische Prinzipienlehre, 230-240, zit. 235, jetzt in: ders., Kirche - Zeichen unter den Völkern, 879-891, zit. 885. 
Augsburg nicht gelungen ist, hat die Geschichte einen wesentlich anderen Lauf genommen als den, der in Augsburg anvisiert gewesen ist. Das vorgenommene Ziel ist auf dem Augsburger Reichstag mit der Verlesung der Confessio Augustana nicht erreicht worden. Augsburg ist der letzte kraftvolle Versuch zur Versöhnung gewesen, der jedoch gescheitert ist, weshalb es nicht zur wirksamen Einheit, sondern zur sichtbaren Trennung der Christenheit im Abendland gekommen ist. Damit hat das Augsburger Bekenntnis geschichtlich eine wesentlich andere Qualität erhalten: Aus einem Bekenntnis, das verfasst worden ist, um in der religiös problemgeladenen Gesellschaft von damals der Wiederherstellung der Einheit und des Friedens zu dienen, ist bald ein Dokument der Spaltung geworden. Das erfreuliche Friedensangebot ist ein unerfreuliches Streitobjekt geworden. Was als gemeinsames Zeugnis aller Christen konzipiert worden ist, ist zu einem Sonderbekenntnis einer neu entstehenden und gerade nicht vorgesehenen Konfessionskirche geworden.

Damit stellt sich erst recht die Frage, welche Confessio Augustana denn nun in katholischer Sicht anerkannt werden soll: das damalige Dokument der Einheit oder das geschichtlich gewordene Dokument der Trennung? Diese Frage verschärft sich nochmals, wenn man bedenkt, dass die Einschätzung der Confessio Augustana die Sicht der Reformation und die ökumenische Aufgabe überhaupt prägt.

Wer in der Confessio Augustana ein Dokument der Einheit und des ehrlichen Willens, die bedrohte Einheit wiederherzustellen, wahrnimmt, sieht in der evangelischen Bewegung im 16. Jahrhundert den berechtigten Versuch der Erneuerung der ganzen Christenheit im Geist des Evangeliums und ist überzeugt, dass es ihr um eine durchgehende Reform der Kirche und nicht um eine Reformation im Sinne der mit ihr schliesslich zerbrochenen Einheit der Kirche gegangen ist, wie der evangelische Ökumeniker Wolfhart Pannenberg immer wieder betont hat: „Luther wollte eine Reform der Gesamtchristenheit; sein Ziel war alles andere als eine lutherische Sonderkirche. " 25 Als lutherischer Theologe hat Pannenberg deshalb in der historischen Tatsache, dass die evangelische Bewegung in der damaligen Zeit nicht ihrer Intention gemäß verwirklicht werden konnte, dass vielmehr von der Katholischen Kirche getrennte Evangelische Kirchen entstanden sind, nicht das „Gelingen“ der Reformation, sondern ihr „Scheitern“, jedenfalls zumindest eine geschichtlich bedingte Notlösung gesehen. ${ }^{26}$ Das wirkliche Gelingen der Reformation hat er demgegenüber in der Überwindung der Spaltungen

25 W. Pannenberg, Problemgeschichte der neueren evangelischen Theologie in Deutschland, Göttingen 1997, 25.

26 W. Pannenberg, Reformation und Einheit der Kirche, in: ders., Ethik und Ekklesiologie. Gesammelte Aufsätze, Göttingen 1977, 254-267, zit. 255. 
und in der Wiederherstellung der Einheit der im Geist des Evangeliums erneuerten Kirche erblickt, so dass es in der ökumenischen Suche nach der Einheit der Christen auch um die - freilich verspätete - Vollendung der Reformation selbst geht.

Wer hingegen in der Confessio Augustana ein Dokument der Trennung und die Basis einer neu entstandenen Kirche wahrnimmt, wird die Überzeugung vertreten, mit der Reformation habe endlich jene Pluralisierung der lateinischen Christenheit begonnen, die in der permanenten Konkurrenz von selbständigen Konfessionskirchen Gestalt gefunden, in der Form des Protestantismus das Christentum modernitätsverträglich gemacht habe und nicht mit einer neuen Einheitssuche wieder in Frage gestellt werden dürfe. Die Reformation und die anschließende Kirchenspaltung werden dann nicht mehr als Schuld und Tragik, sondern als Erfolg und Fortschritt betrachtet. Der evangelische Kirchenhistoriker Christoph Markschies macht jedenfalls darauf aufmerksam, dass man sich in der „Liberalen Theologie“, die vor allem an deutschsprachigen theologischen Fakultäten weit verbreitet sei, mit der Ökumenischen Bewegung schwer tue, „da dort vielfach das reformatorische Christentum für einen kategorial vom übrigen Christentum geschiedenen, neuzeitkonformen eigenen Typus der Religion gehalten wird und nicht - wie häufig in der <Offenbarungstheologie > - für denjenigen Teil der einen, heiligen und allgemeinen Kirche, der durch die Reformation gegangen ist, aber durch vielfältige Gemeinsamkeit und theologische Traditionslinien mit dieser una sancta catholica ecclesia verbunden ist“"27.

Die Beschäftigung mit der Confessio Augustana hat uns damit zur fundamentaleren und für die ökumenische Situation heute bedeutsamen Frage geführt, wie wir heute, und zwar die ökumenischen Partner für sich und gemeinsam, die Reformation betrachten: nach wie vor, wie in der Vergangenheit üblich, als Bruch mit der bisherigen Tradition der Christenheit, mit dem etwas Neues begonnen hat, oder bei aller Erneuerung in einer bleibenden Kontinuität mit der gesamten Tradition der universalen Kirche. Es handelt sich dabei um jene Frage, die mein Vorgänger als Präsident des Päpstlichen Rates zur Förderung der Einheit der Christen, Walter Kardinal Kasper, bereits im Vorausblick auf das Reformationsgedenken an die aus der Reformation hervorgegangenen kirchlichen Gemeinschaften gestellt hat, ob sie die Reformation als „ein neues Paradigma“ wahrnehmen, „das sich durch eine bleibende Grunddifferenz < protestantisch> vom Katholischen abgrenzt“, oder ob sie diese im ökumenischen Sinn als „Reform und

27 Ch. Markschies, Aufbruch oder Katerstimmung? Zur Lage nach dem Reformationsjubiläum, Hamburg 2017, 67. 
Erneuerung der einen universalen Kirche“ verstehen. ${ }^{28}$ Von der Beantwortung dieser Frage hängt nicht nur die Art und Weise ab, wie der ökumenische Dialog der Katholischen Kirche mit den aus der Reformation hervorgegangenen kirchlichen Gemeinschaften weitergehen soll, sondern auch, wie wir Katholiken die Confessio Augustana wertschätzen und in welcher Weise wir sie anerkennen.

\section{Die Confessio Augustana im Licht der ökumenischen Entwicklungen}

Anlässlich des 450. Jahrestags der Verlesung der Confessio Augustana hat Papst Johannes Paul II. die doppelte Feststellung getroffen, „dass damals zwar der Brückenbau nicht gelang, dass aber wichtige Hauptpfeiler der Brücke im Sturm der Zeiten erhalten geblieben sind“29. Diese Feststellung wird durch die intensiven ökumenischen Dialoge, die in den vergangenen dreißig Jahren geführt worden sind, zusätzlich bestätigt. Zu denken ist vor allem an das ökumenische Projekt Lehrverurteilungen - kirchentrennend?, das zum Ergebnis geführt hat, dass die Lehrverurteilungen der Reformationszeit im Wesentlichen den heutigen Partner nicht mehr treffen; ${ }^{30}$ an die Gemeinsame Erklärung zur Rechtfertigungslehre im Jahre 1999, mit der es möglich geworden ist, bei der wohl zentralsten Frage, die im 16. Jahrhundert zur Reformation und anschließend zur Kirchenspaltung geführt hat, einen Konsens in den Grundfragen zu erreichen; und auch an das von der Evangelisch-Lutherisch/Römisch-katholischen Kommission für die Einheit zur Vorbereitung eines gemeinsamen Gedenkens des Beginns der Reformation erarbeitete und veröffentlichte Dokument Vom Konflikt zur Gemeinschaft, in dem weitgehende Konvergenzen über die Lehre von der Rechtfertigung, über das Verhältnis von Heiliger Schrift und Tradition, über Eucharistie und das kirchliche Amt festgestellt werden konnten. ${ }^{31}$ Alle diese ökumenischen Bemühungen be-

28 Kardinal W. Kasper, Ökumenisch von Gott sprechen?, in: I. U. Dalferth/J. Fischer/H.-P. Grosshans (Hg.), Denkwürdiges Geheimnis. Beiträge zur Gotteslehre. Festschrift für Eberhard Jüngel zum 70. Geburtstag, Tübingen 2004, 291-302, zit. 302.

29 Johannes Paul II., Ansprache aus Anlass des 450. Jahrestages der „Confessio Augustana“ am 25. Juni 1980.

30 Vgl. K. Lehmann/W. Pannenberg (Hg.), Lehrverurteilungen - kirchentrennend? Bd. 1: Rechtfertigung, Sakramente und Amt im Zeitalter der Reformation und heute, Freiburg i.B./Göttingen 1986.

31 Vom Konflikt zur Gemeinschaft. Gemeinsames lutherisch-katholisches Reformationsgedenken im Jahr 2017. Bericht der Lutherisch/Römisch-katholischen Kommission für die Einheit, Leipzig/Paderborn 2013. 
stätigen eine breite gemeinsame Glaubensbasis, die bereits in der Confessio Augustana vorliegt.

Im Blick auf die Confessio Augustana werfen neuere ökumenische Unternehmungen freilich auch neue Fragen auf, was nur an einem Beispiel kurz verdeutlicht werden soll. In dem ekklesiologisch grundlegenden Artikel 7 der Confessio Augustana wird die Kirche als „Versammlung der Heiligen“ definiert, „in der das Evangelium rein gelehrt wird und die Sakramente recht verwaltet werden“; und es wird weiter ausgeführt, dass es zur wahren Einheit der Kirche „genug“ ist (satis est), „dass man übereinstimme in der Lehre des Evangeliums und in der Verwaltung der Sakramente“. Bei der ökumenischen Beschäftigung mit der Confessio Augustana hat sich eine breite Übereinkunft ergeben, dass mit dem satis est keineswegs das kirchliche Amt und seine Ordnungsverantwortung für die Einheit der Kirche als unerheblich erklärt wird, dass vielmehr im Gegenteil das kirchliche Amt im siebten Artikel deshalb nicht eigens erwähnt wird, weil es bereits im fünften Artikel behandelt worden ist, und zwar als Predigtamt, das den rechtfertigenden Glauben vermittelt: Damit wir diesen Glauben erlangen, ist das Amt eingesetzt, welches das Evangelium verkündet und die Sakramente darreicht.

Das Amt ist deshalb, wie Vinzenz Pfnür mit Recht hervorhebt, ,auf keinen Fall unter die < von den Menschen eingesetzten Ceremonien> einzuordnen, denn nach CA V <hat Gott das Predigtamt eingesetzt>. Das kirchliche Amt wird in der CA, wie überhaupt in den lutherischen Bekenntnisschriften nicht aus dem allgemeinen Priestertum aller Gläubigen abgeleitet, sondern aus der Sendung und Beauftragung durch Gott. ${ }^{32}$

Von daher fragt man sich, wie sich die Leuenberger Konkordie, die von lutherischen Kirchen unterzeichnet worden ist, zur Confessio Augustana verhält, wenn es in der Konkordie im Basisartikel 2 heißt: „Die Kirche ist allein auf Jesus Christus gegründet, der sie durch die Zuwendung seines Heils in der Verkündigung und in den Sakramenten sammelt und sendet. Nach reformatorischer Einsicht ist darum zur wahren Einheit der Kirche die Übereinstimmung in der rechten Lehre des Evangeliums und in der rechten Verwaltung der Sakramente notwendig und ausreichend.“ Wird hier nicht das satis est in der Confessio Augustana als „notwendig und ausreichend" interpretiert, so dass das Amt nicht mehr als Wesenselement der Kirche betrachtet wird? ${ }^{33}$

32 V. Pfnür, Einig in der Rechtfertigungslehre? Die Rechtfertigungslehre in der Confessio Augustana (1530) und die Stellungnahme der katholischen Kontroverstheologie zwischen 1530 und 1535, Wiesbaden 1970, $378 \mathrm{f}$.

33 In dem an der vierten Vollversammlung der Leuenberger Kirchengemeinschaft im Jahre 1994 angenommenen Dokument Die Kirche Jesu Christi. Der reformatorische Beitrag zum ökumenischen Dialog über die kirchliche Einheit scheint freilich das Amt als Wesenselement nicht ausgeschlossen 


\section{Anerkennung von Kirchengemeinschaft als Ziel}

Mit diesem Beispiel dürfte deutlich sein, dass wir nicht hinter die ökumenische Situation heute zum Text der Confessio Augustana zurückkehren und ihn als historischen Text katholisch anerkennen können, dass vielmehr die seitherigen Entwicklungen in der ökumenischen Diskussion miteinbezogen werden müssen. Damit ist ein Dreifaches impliziert: Es bedeutet erstens, dass die Katholische Kirche die Confessio Augustana nicht einfach als ein eigenes römisch-katholisches Bekenntnis und dogmatisch verbindliches Lehrdokument rezipieren, sondern dass sie die Confessio Augustana als einen legitimen Ausdruck des gemeinsamen katholischen Glaubens annehmen kann. Hinzu kommt zweitens, dass es nicht nur um die Anerkennung eines isolierten Bekenntnistextes gehen kann, sondern dass es um die „Anerkennung der kirchlichen Gemeinschaft, die sich auf diesen Text beruft und sich von ihm her versteht“"34, gehen muss. Und drittens ist damit impliziert, dass das eigentliche Fundament für diese Anerkennung der kirchlichen Gemeinschaft, die natürlich ein wechselseitiger Vorgang und ein spiritueller Prozess sein muss, nicht die Confessio Augustana an sich sein kann, sondern der von ihr von Anfang an geforderte doppelte Rekurs auf die Heilige Schrift und die Glaubensbekenntnisse und die in ihnen enthaltenen christologisch- trinitarischen Grundentscheidungen der Alten Kirche. Wenn ich recht sehe, ist es doch auch bei der Überreichung der Confessio Augustana an Kaiser Karl V. im Jahre 1530 um diese drei inhaltlichen Perspektiven gegangen.

Die Beschäftigung mit der CA ruft uns deshalb das Gebot der ökumenischen Stunde in Erinnerung, weitere Schritte auf die zu erreichende Kirchengemeinschaft hin zu tun. In diesem Zusammenhang ist das im lutherisch-katholischen Dialog in Aussicht gestellte Projekt zu verstehen, einen tieferen Konsens über Kirche, Eucharistie und Amt, und zwar in ihrer unlösbaren Zusammengehörigkeit, zu finden..$^{35}$ Dieses Projekt bezieht sich dabei auf die in der Gemeinsamen

zu sein, da betont wird, dass das ordinierte Amt nach reformatorischem Verständnis „auf einem besonderen Auftrag Christi“ ruht und „zugleich in seinem Dienst mit der ganzen Gemeinde unter dem Wort Gottes“ steht. Dabei handelt es sich zwar um ein Zitat aus den Tampere-Thesen, es wird aber später im eigenen Text des Dokuments wiederholt: Das ordinierte Amt „beruht auf einem besonderen Auftrag Christi, ist aber stets auf das allgemeine Priestertum angewiesen.“ Vgl. Leuenberger Texte. Heft 1: Die Kirche Jesu Christi. Im Auftrag des Exekutivausschusses für die Leuenberger Kirchengemeinschaft hg.v. W. Hüffmeier, Frankfurt a. M. ${ }^{3} 2011,35$.

34 W. Kasper, Bekenntnis und Bekenntnisgemeinschaft in katholischer Sicht, in: Meyer/Schütte, Bekenntnis des einen Glaubens, 22-46, zit. 45.

35 Vgl. K. Koch, Auf dem Weg zur Kirchengemeinschaft. Welche Chance hat eine gemeinsame Erklärung zu Kirche, Eucharistie und Amt?, in: Catholica 69 (2015), 77-94. 
Erklärung zur Rechtfertigungslehre angezeigten Fragen, die weiterer Klärung bedürfen: „sie betreffen unter anderem das Verhältnis von Wort Gottes und kirchlicher Lehre sowie die Lehre von der Kirche, von der Autorität in ihr, von ihrer Einheit, vom Amt und von den Sakramenten, schließlich von der Beziehung zwischen Rechtfertigung und Sozialethik."36

Wir dürfen dabei dankbar feststellen, dass sich bereits verschiedene nationale Dialoge mit dieser Thematik beschäftigen: Der lutherisch-katholische Dialog in den Vereinigten Staaten von Amerika hat bereits eine diesbezügliche Declaration on the way: Church, Eucharist and Ministry vorgelegt; ${ }^{37}$ und auch der nationale Dialog in Finnland hat zu dieser Thematik die umfangreiche Stellungnahme Communion in Growth. Declaration on Church, Eucharist and Ministry publiziert. ${ }^{38}$ Auf diesen hilfreichen Vorarbeiten gilt es aufzubauen. Denn bei diesen Themen sind noch nicht, wie man verschiedentlich aus Deutschland hören kann, bereits alle Probleme von den Theologen gelöst worden, so dass nur noch die Kirchenleitungen am Zuge wären. Zudem braucht es für verbindliche Schritte auf die Kirchengemeinschaft hin auch verbindliche Entscheidungen der jeweiligen Kirchengemeinschaften. Es führen letztlich nur solche Texte - wie die Confessio Augustana - in die Zukunft, die in den jeweiligen Kirchen rezipiert und von ihren Leitungen autoritativ angenommen werden.

Wenn es gelingen könnte, einen verbindlichen Konsens $\mathrm{zu}$ den wichtigen Themen von Kirche, Eucharistie und Amt zu finden, wäre ein wichtiger Schritt auf die sichtbare Kirchengemeinschaft hin vollzogen, die das Ziel allen ökumenischen Bemühens auch im Dialog mit den aus der Reformation hervorgegangenen kirchlichen Gemeinschaften ist und sein muss. Dabei kann die Beschäftigung mit der Confessio Augustana eine wesentliche Hilfe sein. Sie erinnert uns daran, dass wir heute in derselben ökumenischen Pflicht stehen, wie sie 1530 in der Vorrede der Confessio Augustana formuliert worden ist: „damit durch uns alle eine gemeinsame wahre Religion angenommen und gehalten wird und wir so, wie wir alle unter einem Christus stehen und streiten, auch alle in einer Gemeinschaft und Kirche in Einigkeit leben“.

36 Gemeinsame Erklärung zur Rechtfertigungslehre des Lutherischen Weltbundes und der Katholischen Kirche, Nr. 43, in: Dokumente wachsender Übereinstimmung. Sämtliche Berichte und Konsenstexte interkonfessioneller Gespräche auf Weltebene, Bd. 3: 1990 -2001, hg.v. H. Meyer/D. Papandreou/H. J. Urban/L. Vischer, Paderborn/Frankfurt a. M. 2003, 419-441, zit. 430.

37 Committee on Ecumenical and Interreligious Affairs, United States Conference of Catholic Bishops - Evangelical-Lutheran Church in America, Declaration on the Way. Church, Ministry, and Eucharist, Minneapolis 2015.

38 Communion in Growth. Declaration on the Church, Eucharist, and Ministry. A Report from the Lutheran-Catholic Dialogue-Commission for Finland, Helsinki 2017. 